\title{
Some notes on almost paracomplex structures associated with the diagonal lifts and operators on cotangent bundle $T^{*}\left(M^{n}\right)$
}

\author{
Hasim Cayir and Kubra Akdag \\ Department of Mathematics, Faculty of Arts and Sciences, Giresun University, Giresun, Turkey
}

Received: 22 August 2016, Accepted: 22 September 2016

Published online: 9 October 2016.

\begin{abstract}
In this paper firstly, the Tachibana operators were applied to 1 -form, vertical, complete and horizontal lifts with respect to almost paracomplex structure $I^{D}$ (The diagonal lift $I^{D}$ ) on cotangent bundle. Secondly, the Vishnevskii operators were applied to 1 -form according to the diagonal lift $I^{D}$ on cotangent bundle. Finally, covariant derivatives of almost paracomplex structure $I^{D}$ with respect to vertical, complete and horizontal lifts were obtained.
\end{abstract}

Keywords: Tachibana Operators, Vishnevskii Operators, Almost Paracomplex Structure, Vertical Lift, Horizontal Lift, Diagonal Lift

\section{Introduction}

The tangent bundles of differentiable manifolds are very important in many areas of mathematics, physics and different topics of geometry (see $[3,13,14,17,26]$ for curves etc.). The geometry of tangent bundles goes back to the fundamental paper[24] of Sasaki published in 1958. Cotangent bundle is dual of the tangent bundle. Because of this duality, some of the geometric results are similar to each other. The most significant difference between them is construction of lifts (see [27] for more details). In 1952, para-complex manifolds, almost para-Hermitian manifolds and para-Kähler manifolds were defined by Libermann[15] (see [1,11,10,25] for Kähler manifolds, Kähler-Norden manifolds and almost complex manifolds). In 1985, Kaneyuki and Williams defined the almost para-contact structure on a pseudo-Riemannian manifold $M$ of dimension $2 m+1$ and constructed the almost para-complex structure on $M^{2 m+1} \times R$ [12].

Let $M^{n}$ be an $n$-dimensional Riemannian manifold of class $C^{\infty}$ and with metric $g, T^{*} M^{n}$ its cotangent bundle and $\pi$ the natural projection $T^{*} M^{n} \rightarrow M^{n}$. A system of local coordinates $\left(U, x^{i}\right), i=1, \ldots, n$ in $M^{n}$ induces on $T^{*} M^{n}$. A system of local coordinates $\left(\pi^{-1}(U), x^{i}, x^{\bar{l}}=p_{i}\right), \bar{l}:=n+i=n+1, \ldots, 2 n$, where $x^{\bar{l}}=p_{i}$ is the component of covectors $p$ in each cotangent space $T_{x}^{*} M^{n}, x \in U$ with respect to the natural coframe $\left\{d x^{i}\right\}$.

We denote by $\mathfrak{I}_{s}^{r}\left(M^{n}\right)\left(\mathfrak{I}_{s}^{r}\left(T^{*} M^{n}\right)\right)$ the module over $F\left(M^{n}\right)\left(F\left(T^{*} M^{n}\right)\right)$ of $C^{\infty}$ tensor fields of type $(r, s)$, where $F\left(M^{n}\right)\left(F\left(T^{*} M^{n}\right)\right)$ is the ring of real-valued $C^{\infty}$ functions on $M^{n}\left(T^{*} M^{n}\right)$.

Let $X=X^{i} \frac{\partial}{\partial x^{i}}$ and $\omega=\omega_{i} d x^{i}$ be the local expressions in $U \subset M^{n}$ of a vector and a covector (1-form) fields $X \in \mathfrak{I}_{0}^{1}\left(M^{n}\right)$ and $\omega \in \mathfrak{I}_{1}^{0}\left(M^{n}\right)$, respectively. Then the complete and horizontal lifts $X^{C}, X^{H} \in \mathfrak{I}_{0}^{1}\left(T^{*} M^{n}\right)$ of $X \in \mathfrak{I}_{0}^{1}\left(M^{n}\right)$ and the 
vertical lift $\omega^{V} \in \mathfrak{I}_{0}^{1}\left(T^{*} M^{n}\right)$ of $\omega \in \mathfrak{I}_{1}^{0}\left(M^{n}\right)$ are given, respectively by

$$
\begin{aligned}
X^{C} & =X^{i} \frac{\partial}{\partial x^{i}}-\sum_{i} p_{h} \partial_{i} X^{h} \frac{\partial}{\partial x^{\bar{l}}} \\
X^{H} & =X^{i} \frac{\partial}{\partial x^{i}}-\sum_{i} p_{h} \Gamma_{i j}^{h} X^{j} \frac{\partial}{\partial x^{\bar{l}}} \\
\omega^{V} & =\sum_{i} w_{i} \frac{\partial}{\partial x^{\bar{l}}}
\end{aligned}
$$

with respect to the natural frame $\left\{\frac{\partial}{\partial x^{i}}, \frac{\partial}{\partial x^{i}}\right\}$, where $\Gamma_{i j}^{h}$ are the components of the Levi-Civita connection $\nabla_{g}$ on $M^{n}$ [19, 27].

Definition 1. Let $M^{n}$ be an $n$-dimensional differentiable manifold. Differantial transformation $D=L_{X}$ is called Lie derivation with respect to vector field $X \in \mathfrak{I}_{0}^{1}\left(M^{n}\right)$ if

$$
\begin{aligned}
& L_{X} f=X f, \forall f \in \mathfrak{I}_{0}^{0}\left(M^{n}\right), \\
& L_{X} Y=[X, Y], \forall X, Y \in \mathfrak{I}_{0}^{1}\left(M^{n}\right) .
\end{aligned}
$$

$[X, Y]$ is called by Lie bracked. The Lie derivative $L_{X} F$ of a tensor field $F$ of type $(1,1)$ with respect to a vector field $X$ is defined by $[5,8,27]$

$$
\left(L_{X} F\right) Y=[X, F Y]-F[X, Y]
$$

Definition 2. Let $M^{n}$ be an $n$-dimensional differentiable manifold. Differantial transformation of algebra $T\left(M^{n}\right)$, defined by

$$
D=\nabla_{X}: T\left(M^{n}\right) \rightarrow T\left(M^{n}\right), X \in \mathfrak{I}_{0}^{1}\left(M^{n}\right),
$$

is called as covariant derivation with respect to vector field $X$ if

$$
\begin{aligned}
\nabla_{f X+g Y} t & =f \nabla_{X} t+g \nabla_{Y} t, \\
\nabla_{X} f & =X f,
\end{aligned}
$$

where $\forall f, g \in \mathfrak{I}_{0}^{0}\left(M^{n}\right), \forall X, Y \in \mathfrak{I}_{0}^{1}\left(M^{n}\right), \forall t \in \mathfrak{I}\left(M^{n}\right)$.

On the other hand, a transformation defined by

$$
\nabla: \mathfrak{I}_{0}^{1}\left(M^{n}\right) \times \mathfrak{I}_{0}^{1}\left(M^{n}\right) \rightarrow \mathfrak{I}_{0}^{1}\left(M^{n}\right)
$$

is called as an affine connection[22,27].

Proposition 1. Covariant differentiation with respect to the complete lift $\nabla^{C}$ of a symetric affine connection $\nabla$ in $M^{n}$ to $T^{*}\left(M^{n}\right)$ has the following properties:

$$
\begin{aligned}
\nabla_{\omega^{V}}^{C} \theta^{V} & =0, \nabla_{\omega^{V}}^{C} Y^{C}=-\gamma(\omega o(\nabla Y))=-(p(\omega o(\nabla Y)))^{V}, \nabla_{X^{C}}^{C} \theta^{V}=\left(\nabla_{X} \theta\right)^{V}, \\
\nabla_{X}^{C}{ }^{C} Y^{C} & =\left(\nabla_{X} Y\right)^{C}+\gamma\left(\nabla\left(\nabla_{X} Y+\nabla_{Y} X\right)\right)-\gamma\left(\nabla_{X} \nabla Y+\nabla_{Y} \nabla X\right) \\
& =\left(\nabla_{X} Y\right)^{C}+\left(p\left(\left(\nabla\left(\nabla_{X} Y+\nabla_{Y} X\right)\right)-\left(\nabla_{X} \nabla Y+\nabla_{Y} \nabla X\right)\right)\right)^{V}
\end{aligned}
$$

for $X, Y \in \mathfrak{I}_{0}^{1}\left(M^{n}\right), \theta, \omega \in \mathfrak{I}_{1}^{0}\left(M^{n}\right)[27]$. 
Proposition 2. Covariant differentiation with respect to the horizontal lift $\nabla^{H}$ of a symetric affine connection $\nabla$ in $M^{n}$ to $T^{*}\left(M^{n}\right)$ satisfies

$$
\begin{aligned}
\nabla_{X^{H}}^{H} Y^{H} & =\left(\nabla_{X} Y\right)^{H}, \nabla_{\theta^{V}}^{H} \omega^{V}=0, \\
\nabla_{X^{H}}^{H} \omega^{V} & =\left(\nabla_{X} \omega\right)^{V}, \nabla_{\theta^{V}}^{H} Y^{H}=0,
\end{aligned}
$$

for any $X, Y \in \mathfrak{I}_{0}^{1}\left(M^{n}\right), \theta, \omega \in \mathfrak{I}_{1}^{0}\left(M^{n}\right)[27]$.

\subsection{An special type of almost paracomplex structure (the diagonal lift $I^{D}$ ) in the cotangent bundle}

An almost paracomplex manifold is an almost product manifold $\left(M^{n}, \varphi\right), \varphi^{2}=I$, such that the two eigenbundles $T^{+} M^{n}$ and $T^{-} M^{n}$ associated to the two eigenvalues +1 and -1 of $\varphi$, respectively, have the same rank. Note that the dimension of an almost paracomplex manifold is necessarly even. Considering the paracomplex structure $\varphi$, we obtain the set $\{I, \varphi\}$ on $M^{n}$, which is an isomorphic representation algebra of order 2, which is called the algebra of paracomplex (or double) numbers and is denoted by $R(j), j^{2}=1$. A tensor field $\omega \in \mathfrak{I}_{q}^{0}\left(M^{2 n}\right)$ is said to be a pure with respect to the paracomplex structure $\varphi$, if

$$
\omega\left(\varphi X_{1}, X_{2}, \ldots, X_{q}\right)=\omega\left(X_{1}, \varphi X_{2}, \ldots, X_{q}\right)=\omega\left(X_{1}, X_{2}, \ldots, \varphi X_{q}\right)
$$

for any $X_{1}, X_{2}, \ldots, X_{q} \in \mathfrak{I}_{0}^{1}\left(M^{2 n}\right)$. We define the operator $\phi_{\varphi}$ associated with $\varphi$ and apply to the pure tensor field $\omega$ :

$$
\begin{aligned}
\left(\phi_{\varphi} \omega\right)\left(Y, X_{1}, X_{2}, \ldots, X_{q}\right) & =(\varphi Y)\left(\omega\left(X_{1}, X_{2}, \ldots, X_{q}\right)\right)-Y\left(\omega\left(\varphi X_{1}, X_{2}, \ldots, X_{q}\right)\right) \\
& +\omega\left(\left(L_{X_{1}} \varphi\right) Y, X_{2}, \ldots, X_{q}\right)+\cdots+\omega\left(X_{1}, X_{2}, \ldots,\left(L_{X q} \varphi\right) Y\right)
\end{aligned}
$$

where $L_{X}$ denotes the Lie derivative with respect to $X$. We note that $\phi_{\varphi} \omega \in \mathfrak{I}_{q+1}^{0}\left(M^{2 n}\right)$. If $\phi_{\varphi} \omega=0$, then $\omega$ is said to be almost paraholomorphic with respect to the paracomplex algebra $R(j)$ (see $[4,19,22]$ ).

Definition 3. In a manifold with almost paracomplex structure $\varphi$, a vector field $X$ is called an almost paraholomorphic vector field if $L_{X} \varphi=0$.

Let $F \in \mathfrak{I}_{1}^{1}\left(M^{n}\right)$. We define a tensor field $F^{D}$ of type $(1,1)$ in $T^{*} M^{n}$ by

$$
F^{D} X^{H}=(F X)^{H}, F^{D} \omega^{V}=-(\omega o F)^{V}=-(\omega F)^{V}
$$

for any $X \in \mathfrak{I}_{0}^{1}\left(M^{n}\right)$ and $\omega \in \mathfrak{I}_{1}^{0}\left(M^{n}\right)$. We call $F^{D}$ the diagonal lift of the tensor field $F$. $F^{D}$ has components

$$
F^{D}=\left(\begin{array}{cc}
F_{i}^{h} & 0 \\
0 & -F_{h}^{i}
\end{array}\right)
$$

with respect to the adapted frame $\tilde{e}_{(\alpha)}$. The diagonal lift $I^{D}$ of identity tensor field $I$ of type $(1,1)$ has the components

$$
I^{D}=\left(\begin{array}{cc}
\delta_{j}^{i} & 0 \\
2 p_{a} \Gamma_{i j}^{a} & -\delta_{j}^{i}
\end{array}\right)
$$

with respect to the induced coordinates and satisfies $\left(I^{D}\right)^{2}=I$. Thus $I^{D}$ is an almost product structure determining the horizontal distribution and the distribution consisting of the tangent planes to fibres [27]. If we put $F^{D}=I^{D}$ in (10) for all vector fields $\tilde{X}$ and $\tilde{Y}$ which are of the form $\omega^{V}, \theta^{V}$ or $X^{H}, Y^{H}$, then we get

$$
I^{D} \omega^{V}=-\omega^{V}, I^{D} X^{H}=X^{H}
$$


Theorem 1. $\left(T^{*} M^{n}, I^{D}, S\right)$ is an almost paracomplex Riemannian manifold.

It is well known that (p. 238, p. 277 of [27])

$$
\begin{aligned}
& { }^{V} \omega^{V} f=0,{ }^{H} X^{V} f={ }^{V}(X f) \\
& {\left[{ }^{H} X,{ }^{V} \omega\right]={ }^{V}\left(\nabla_{X} \omega\right),\left[{ }^{V} \omega,{ }^{V} \theta\right]=0} \\
& {\left[{ }^{H} X,{ }^{H} Y\right]={ }^{H}[X, Y]+\gamma R(X, Y)={ }^{H}[X, Y]+{ }^{V}(p R(X, Y))} \\
& p R(X, Y)=\left(p_{i}\left(R(X, Y){ }_{j}^{i}\right)\right) \text { and } \\
& I^{D}(p R(X, Y))^{V}=-(p R(X, Y))^{V},
\end{aligned}
$$

where $R$ is the curvature tensor of $\nabla$ and the term involving the Lie derivative on the connection $\nabla$ is given by $\left(L_{X} \nabla\right)_{Y}=$ $\nabla_{Y} \nabla X+R(X, Y), Y$ being an arbitrary element of $\mathfrak{I}_{0}^{1}\left(M^{n}\right)$

\section{Main results}

2.1 The Tachibana operators applied to 1-form, vertical, complete and horizontal lifts with respect to almost paracomplex structure $I^{D}$

Definition 4. Let $\varphi \in \mathfrak{I}_{1}^{1}\left(M^{n}\right)$, and $\mathfrak{I}\left(M^{n}\right)=\sum_{r, s=0}^{\infty} \mathfrak{I}_{s}^{r}\left(M^{n}\right)$ be a tensor algebra over $R$. A map $\left.\phi_{\varphi}\right|_{r+s) 0}: \mathfrak{J}^{*}\left(M^{n}\right) \rightarrow \mathfrak{I}\left(M^{n}\right)$ is called a Tachibana operator or $\phi_{\varphi}$ operator on $M^{n}$ if

(a) $\phi_{\varphi}$ is linear with respect to constant coefficient,

(b) $\phi_{\varphi}: \stackrel{*}{\mathfrak{I}}\left(M^{n}\right) \rightarrow \mathfrak{I}_{s+1}^{r}\left(M^{n}\right)$ for all $r$ and $s$,

(c) $\phi_{\varphi}\left(K^{C} \otimes\right)=\left(\phi_{\varphi} K\right) \otimes L+K \otimes \phi_{\varphi} L$ for all $K, L \in \stackrel{*}{\mathfrak{I}}\left(M^{n}\right)$,

(d) $\phi_{\varphi X} Y=-\left(L_{Y} \varphi\right) X$ for all $X, Y \in \mathfrak{I}_{0}^{1}\left(M^{n}\right)$ where $L_{Y}$ is the Lie derivation with respect to $Y$,

(e) $\left(\phi_{\varphi X} \eta\right) Y=\left(d\left(l_{Y} \eta\right)\right)(\varphi X)-\left(d\left(l_{Y}(\eta \circ \varphi)\right)\right) X+\eta\left(\left(L_{Y} \varphi\right) X\right)=\phi X\left(l_{Y} \eta\right)-X\left(l_{\varphi Y} \eta\right)+\eta\left(\left(L_{Y} \varphi\right) X\right)$

for all $\eta \in \mathfrak{I}_{1}^{0}\left(M^{n}\right)$ and $X, Y \in \mathfrak{I}_{0}^{1}\left(M^{n}\right)$, where $\iota_{Y} \eta=\eta(Y)=\eta \stackrel{C}{\otimes} Y, \mathfrak{I}_{s}^{*}\left(M^{n}\right)$ the module of all pure tensor fields of type $(r, s)$ on $M^{n}$ according to the affinor field $\varphi[4,6,7,22]$ (see [23] for applied to pure tensor field).

Theorem 2. For $L_{X}$ the operator Lie derivation with respect to $X$, an almost paracomplex structure $I^{D} \in \mathfrak{I}_{1}^{1}\left(T^{*}\left(M^{n}\right)\right)$ defined by (11), $\phi_{I^{D}}$ the Tachibana operator on $M^{n}$, we get the following formulas

(i) $\left(\phi_{\mathrm{I}^{\mathrm{D}}} \omega\right)\left(\mathrm{X}^{\mathrm{H}}, \mathrm{Y}^{\mathrm{H}}\right)=2 \omega(p R(Y, X))^{V}$,

(ii) $\left(\phi_{\mathrm{I}_{\mathrm{D}}} \omega\right)\left(\mathrm{X}^{\mathrm{H}}, \theta^{\mathrm{V}}\right)=2 X^{H}\left(\omega\left(\theta^{V}\right)\right)-2 \omega\left(\nabla_{X} \theta\right)^{V}$

(iii) $\left(\phi_{\mathrm{I}^{\mathrm{D}}} \omega\right)\left(\theta^{\mathrm{V}}, \mathrm{Y}^{\mathrm{H}}\right)=-2 \theta^{V}\left(\omega\left(Y^{H}\right)\right)$,

(iv) $\left(\phi_{\mathrm{I}} \mathrm{D} \omega\right)\left(\theta^{\mathrm{V}}, \xi^{\mathrm{V}}\right)=0$,

where $R$ is the curvature tensor of $\nabla, X^{H}, Y^{H} \in \mathfrak{I}_{0}^{1}\left(T^{*}\left(M^{n}\right)\right)$ of $X \in \mathfrak{I}_{0}^{1}\left(M^{n}\right)$ and the vertical lift $\xi^{V}, \theta^{V} \in \mathfrak{I}_{0}^{1}\left(T^{*}\left(M^{n}\right)\right)$ of $\xi, \theta \in \mathfrak{I}_{1}^{0}\left(M^{n}\right)$. 
Proof. From (11) and (13), we have

(i) $\left(\phi_{\mathrm{I}^{\mathrm{D}}} \omega\right)\left(\mathrm{X}^{\mathrm{H}}, \mathrm{Y}^{\mathrm{H}}\right)=\left(\phi_{I^{D} X^{H}} \omega\right) Y^{H}$

$$
\begin{aligned}
& =I^{D} X^{H}\left(\omega\left(Y^{H}\right)-X^{H}\left(\omega\left(I^{D} Y^{H}\right)\right)+\omega\left(\left(L_{Y^{H}} I^{D}\right) X^{H}\right)\right. \\
& =X^{H}\left(\omega Y^{H}\right)-X^{H}\left(\omega Y^{H}\right)+\omega\left(\left[Y^{H}, I^{D} X^{H}\right]-I^{D}\left[Y^{H}, X^{H}\right]\right) \\
& =\omega\left(\left[Y^{H}, X^{H}\right]-I^{D}\left[Y^{H}, X^{H}\right]\right) \\
& =\omega\left(\left([Y, X]^{H}+(p R(Y, X))^{V}\right)-I^{D}\left([Y, X]^{H}+(p R(Y, X))^{V}\right)\right) \\
& =\omega\left([Y, X]^{H}+(p R(Y, X))^{V}-[Y, X]^{H}-I^{D}(p R(Y, X))^{V}\right) \\
& =\omega\left((p R(Y, X))^{V}+(p R(Y, X))^{V}\right)=2 \omega(p R(Y, X))^{V}
\end{aligned}
$$

(ii) $\left(\phi_{\mathrm{I}^{\mathrm{D}}} \omega\right)\left(\mathrm{X}^{\mathrm{H}}, \theta^{\mathrm{V}}\right)=\left(\phi_{I^{D} X^{H}} \omega\right) \theta^{V}$

$$
\begin{aligned}
& =I^{D} X^{H}\left(\omega\left(\theta^{V}\right)\right)-X^{H}\left(\omega\left(I^{D} \theta^{V}\right)\right)+\omega\left(\left(L_{\theta^{V}} I^{D}\right) X^{H}\right) \\
& =X^{H}\left(\omega\left(\theta^{V}\right)\right)+X^{H}\left(\omega\left(\theta^{V}\right)\right)+\omega\left(\left(L_{\theta^{V}} I^{D} X^{H}-I^{D} L_{\theta^{V}} X^{H}\right)\right. \\
& =2 X^{H}\left(\omega\left(\theta^{V}\right)\right)+\omega\left(\left[\theta^{V}, X^{H}\right]-I^{D}\left[\theta^{V}, X^{H}\right]\right) \\
& =2 X^{H}\left(\omega\left(\theta^{V}\right)\right)+\omega\left(-\left(\nabla_{X} \theta\right)^{V}+I^{D}\left(\nabla_{X} \theta\right)^{V}\right) \\
& =2 X^{H}\left(\omega\left(\theta^{V}\right)\right)+\omega\left(-\left(\nabla_{X} \theta\right)^{V}-\left(\nabla_{X} \theta\right)^{V}\right) \\
& =2 X^{H}\left(\omega\left(\theta^{V}\right)\right)-2 \omega\left(\nabla_{X} \theta\right)^{V}
\end{aligned}
$$

(iii) $\left(\phi_{\mathrm{I}^{\mathrm{D}}} \omega\right)\left(\theta^{\mathrm{V}}, \mathrm{Y}^{\mathrm{H}}\right)=\left(\phi_{I^{D} \theta^{V}} \omega\right) Y^{H}$

$$
\begin{aligned}
& =I^{D} \theta^{V}\left(\omega\left(Y^{H}\right)\right)-\theta^{V}\left(\omega\left(I^{D} Y^{H}\right)\right)+\omega\left(\left(L_{Y^{H}} I^{D}\right) \theta^{V}\right) \\
& =-\theta^{V}\left(\omega\left(Y^{H}\right)\right)-\theta^{V}\left(\omega\left(Y^{H}\right)\right)+\omega\left(\left[Y^{H}, I^{D} \theta^{V}\right]-I^{D}\left[Y^{H}, \theta^{V}\right]\right) \\
& =-2 \theta^{V}\left(\omega\left(Y^{H}\right)\right)+\omega\left(-\left[Y^{H}, \theta^{V}\right]-I^{D}\left[Y^{H}, \theta^{V}\right]\right) \\
& =-2 \theta^{V}\left(\omega\left(Y^{H}\right)\right)+\omega\left(-\left(\nabla_{Y} \theta\right)^{V}-I^{D}\left(\nabla_{Y} \theta\right)^{V}\right) \\
& =-2 \theta^{V}\left(\omega\left(Y^{H}\right)\right)+\omega\left(-\left(\nabla_{Y} \theta\right)^{V}+\left(\nabla_{Y} \theta\right)^{V}\right)=-2 \theta^{V}\left(\omega\left(Y^{H}\right)\right)
\end{aligned}
$$

(iv) $\left(\phi_{\mathrm{I}^{\mathrm{D}}} \omega\right)\left(\theta^{\mathrm{V}}, \xi^{\mathrm{V}}\right)=\left(\phi_{I^{D} \theta^{V}} \omega\right) \xi^{V}$

$$
\begin{aligned}
& =I^{D} \theta^{V}\left(\omega\left(\xi^{V}\right)\right)-\theta^{V}\left(\omega\left(I^{D} \xi^{V}\right)+\omega\left(L_{\xi^{V}} I^{D}\right) \theta^{V}\right. \\
& =-\theta^{V}\left(\omega\left(\xi^{V}\right)\right)+\theta^{V}\left(\omega\left(\xi^{V}\right)\right)+\omega\left(\left[\xi^{V}, I^{D} \theta^{V}\right]-I^{D}\left[\xi^{V}, \theta^{V}\right]\right) \\
& =\omega\left(\left[\xi^{V},-\theta^{V}\right]-I^{D}\left[\xi^{V}, \theta^{V}\right]\right)=\omega\left(-\left[\xi^{V}, \theta^{V}\right]-I^{D}\left[\xi^{V}, \theta^{V}\right]=0\right.
\end{aligned}
$$

Theorem 3. For an almost paracomplex structure $I^{D} \in \mathfrak{I}_{1}^{1}\left(T^{*}\left(M^{n}\right)\right)$ defined by (11), $\phi_{I^{D}}$ the Tachibana operator on $M^{n}$, we get the following results

(i) $\phi_{\mathrm{I}^{\mathrm{D}} \theta^{\mathrm{v}}} \mathrm{X}^{\mathrm{C}}=0$,

(ii) $\phi_{\mathrm{I}^{\mathrm{D}} \mathrm{Y}^{\mathrm{H}}} \theta^{\mathrm{V}}=2\left(\nabla_{Y} \theta\right)^{V}$,

(iii) $\phi_{\mathrm{I}^{\mathrm{D}} \theta^{\mathrm{v}}} \mathrm{X}^{\mathrm{H}}=0$,

(iv) $\phi_{\mathrm{I}^{\mathrm{D}} \theta^{\vee}} \xi^{\mathrm{V}}=0$

(v) $\phi_{\mathrm{I}} \mathrm{Y}_{\mathrm{H}} \mathrm{X}^{\mathrm{H}}=-2\left(p(R(X, Y))^{V}\right.$,

(vi) $\phi_{\mathrm{I}^{\mathrm{D}} \mathrm{Y}^{\mathrm{H}}} \mathrm{X}^{\mathrm{C}}=-2\left(p\left(L_{X} \nabla\right)_{Y}\right)^{V}$,

where $L_{X}$ the operator Lie derivation with respect to $X, X^{C}, X^{H} \in \mathfrak{I}_{0}^{1}\left(T^{*}\left(M^{n}\right)\right)$ of $X \in \mathfrak{I}_{0}^{1}\left(M^{n}\right)$ and the vertical lift $\xi^{V}$, $\theta^{V}, \omega^{V} \in \mathfrak{I}_{0}^{1}\left(T^{*}\left(M^{n}\right)\right)$ of $\xi, \theta, \omega \in \mathfrak{I}_{1}^{0}\left(M^{n}\right)$. 
Proof. From (5), (11), (12) and definition 4, we have

(i) $\phi_{\mathrm{I}^{\mathrm{D}} \theta^{\mathrm{v}}} \mathrm{X}^{\mathrm{C}}=-\left(L_{X^{C}} I^{D}\right) \theta^{V}=-\left(L_{X^{C}} I^{D} \theta^{V}-I^{D} L_{X^{C}} \theta^{V}\right)=-\left(-L_{X^{C}} \theta^{V}-I^{D} L_{X^{C}} \theta^{V}\right)$

$$
=\left(L_{X} \theta\right)^{V}+I^{D}\left(L_{X} \theta\right)^{V}=\left(L_{X} \theta\right)^{V}-\left(L_{X} \theta\right)^{V}=0
$$

(ii) $\phi_{\mathrm{I}^{\mathrm{D} Y^{\mathrm{H}}}} \theta^{\mathrm{V}}=-\left(L_{\theta^{V}} I^{D}\right) Y^{H}=-\left(L_{\theta^{V}} I^{D} Y^{H}-I^{D} L_{\theta^{V}} Y^{H}\right)=-L_{\theta^{V}} Y^{H}+I^{D} L_{\theta^{V}} Y^{H}$

$$
=-\left(-\left(\nabla_{Y} \theta\right)^{V}\right)+I^{D}\left(-\left(\nabla_{Y} \theta\right)^{V}\right)=\left(\nabla_{Y} \theta\right)^{V}-I^{D}\left(\nabla_{Y} \theta\right)^{V}=2\left(\nabla_{Y} \theta\right)^{V}
$$

(iii) $\left(\phi_{\mathrm{I}^{\mathrm{D}} \theta^{\mathrm{v}}} \mathrm{X}^{\mathrm{H}}\right)=-\left(L_{X^{H}} I^{D}\right) \theta^{V}=-\left(L_{X^{H}} I^{D} \theta^{V}-I^{D} L_{X^{H}} \theta^{V}\right)$

$$
\begin{aligned}
& =-\left(-L_{X^{H}} \theta^{V}-I^{D}\left(\nabla_{X} \theta\right)^{V}\right)=-\left(-\left(\nabla_{X} \theta\right)^{V}+\left(\nabla_{X} \theta\right)^{V}\right) \\
& =\left(\nabla_{X} \theta\right)^{V}-\left(\nabla_{X} \theta\right)^{V}=0
\end{aligned}
$$

(iv) $\left(\phi_{\mathrm{I}^{\mathrm{D}} \theta^{\mathrm{v}}} \xi^{\mathrm{V}}\right)=-\left(L_{\xi^{V}} I^{D}\right) \theta^{V}=-\left(L_{\xi^{V}} I^{D} \theta^{V}-I^{D} L_{\xi^{V}} \theta^{V}\right)$

$$
=-\left(-L_{\xi^{V}} \theta^{V}-I^{D} L_{\xi^{V}} \theta^{V}\right)=L_{\xi^{V}} \theta^{V}-I^{D} L_{\xi^{V}} \theta^{V}=0
$$

(v) $\phi_{\mathrm{I}^{\mathrm{D}} \mathrm{Y}^{\mathrm{H}}} \mathrm{X}^{\mathrm{H}}=-\left(L_{X^{H}} I^{D}\right) Y^{H}=-\left(L_{X^{H}} I^{D} Y^{H}-I^{D} L_{X^{H}} Y^{H}\right)=-L_{X^{H}} Y^{H}+I^{D} L_{X^{H}} Y^{H}$

$$
\begin{aligned}
& =-\left([X, Y]^{H}+(p R(X, Y))^{V}\right)+I^{D}\left([X, Y]^{H}+(p R(X, Y))^{V}\right) \\
& =-[X, Y]^{H}-(p R(X, Y))^{V}+[X, Y]^{H}-(p R(X, Y))^{V}=-2\left(p(R(X, Y))^{V}\right.
\end{aligned}
$$

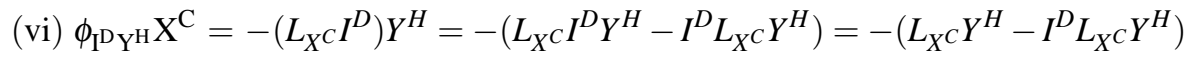

$$
\begin{aligned}
& =-\left([X, Y]^{H}+\left(p\left(L_{X} \nabla\right)_{Y}\right)^{V}-I^{D}\left([X, Y]^{H}+\left(p\left(L_{X} \nabla\right)_{Y}\right)^{V}\right)\right. \\
& =-[X, Y]^{H}-\left(p\left(L_{X} \nabla\right)_{Y}\right)^{V}+I^{D}[X, Y]^{H}+I^{D}\left(p\left(L_{X} \nabla\right)_{Y}\right)^{V} \\
& =-[X, Y]^{H}-\left(p\left(L_{X} \nabla\right)_{Y}\right)^{V}+[X, Y]^{H}-\left(p\left(L_{X} \nabla\right)_{Y}\right)^{V}=-2\left(p\left(L_{X} \nabla\right)_{Y}\right)^{V}
\end{aligned}
$$

2.2 The Vishnevskii Operators applied to 1-form and covarient derivatives of almost paracomplex structure $I^{D}$ with respect to vertical, complete and horizontal lifts

Definition 5. Suppose now that $\nabla$ is a linear connection on $M^{n}$, and let $\varphi \in \mathfrak{I}_{1}^{1}\left(M^{n}\right)$. We can replace the condition d) of defination 4 by

$$
\left.d^{\prime}\right) \psi_{\varphi X} Y=\nabla_{\varphi X} Y-\varphi \nabla_{X} Y
$$

for any $X, Y \in \mathfrak{I}_{0}^{1}\left(M^{n}\right)$. Then we can consider a new operator by a Vishnevskii operator or $\psi_{\varphi}$-operator on $M^{n}$, we shall mean a map $\psi_{\varphi}: \stackrel{*}{\mathfrak{I}}\left(M^{n}\right) \rightarrow \mathfrak{I}\left(M^{n}\right)$, which satisfies conditions $\left.\left.\left.\left.a\right), b\right), c\right), e\right)$ of definition 4 and the condition $\left(d^{\prime}\right)[6,7$, 22] (see $[9,18]$ for different type operators).

Let $\omega \in \mathfrak{I}_{1}^{0}(M)$. Using Definition 5, we have

$$
\begin{aligned}
\left(\psi_{\varphi} \omega\right)(X, Y) & =\left(\psi_{\varphi X} \omega\right) Y \\
& =(\varphi X)\left(\imath_{Y} \omega\right)-X\left(\imath_{\varphi Y} \omega\right)-\omega\left(\nabla_{\varphi X} Y-\varphi\left(\nabla_{X} Y\right)\right) \\
& =\left(\nabla_{\varphi X} \omega-\nabla_{X}(\omega \circ \varphi)\right) Y
\end{aligned}
$$


for any $X, Y \in \mathfrak{I}_{0}^{1}(M)$, where $(\omega \circ \varphi) Y=\omega(\varphi Y)$. From (16) we see that $\psi_{\varphi X} \omega=\nabla_{\varphi X} \omega-\nabla_{X}(\omega \circ \varphi)$ is a 1-form[22].

Theorem 4. For an almost paracomplex structure $I^{D} \in \mathfrak{I}_{1}^{1}\left(T^{*}\left(M^{n}\right)\right)$ defined by $(11), \psi_{I^{D}}$ the Vishnevskii operator on $M^{n}$, we get the following results

(i) $\left(\psi_{\mathrm{I}^{\mathrm{D}}} \omega\right)\left(\mathrm{X}^{\mathrm{H}}, \mathrm{Y}^{\mathrm{H}}\right)=0$,

(ii) $\left(\psi_{\mathrm{I}^{\mathrm{D}}} \omega\right)\left(\mathrm{X}^{\mathrm{H}}, \theta^{\mathrm{V}}\right)=2 X^{H}\left(\omega\left(\theta^{V}\right)\right)-2 \omega\left(\nabla_{X} \theta\right)^{V}$,

(iii) $\left(\psi_{\mathrm{I}^{\mathrm{D}}} \omega\right)\left(\theta^{\mathrm{V}}, \mathrm{Y}^{\mathrm{H}}\right)=-2 \theta^{V}\left(\omega\left(Y^{H}\right)\right)$,

(iv) $\left(\psi_{\mathrm{I}^{\mathrm{D}}} \omega\right)\left(\theta^{\mathrm{V}}, \xi^{\mathrm{V}}\right)=0$,

where the horizontal lift $\nabla^{H}$ of an affine connection $\nabla$ in $M^{n}$ to $T^{*}\left(M^{n}\right), X^{C}, X^{H} \in \mathfrak{I}_{0}^{1}\left(T^{*}\left(M^{n}\right)\right)$ of $X \in \mathfrak{I}_{0}^{1}\left(M^{n}\right)$ and the vertical lift $\xi^{V}, \theta^{V}, \omega^{V} \in \mathfrak{I}_{0}^{1}\left(T^{*}\left(M^{n}\right)\right)$ of $\xi, \theta, \omega \in \mathfrak{I}_{1}^{0}\left(M^{n}\right)$.

Proof. From definition 5 and (16), we get

(i) $\left(\psi_{I^{\mathrm{D}}} \omega\right)\left(\mathrm{X}^{\mathrm{H}}, \mathrm{Y}^{\mathrm{H}}\right)=\left(\psi_{I^{D} X^{H}} \omega\right) Y^{H}=I^{D} X^{H}\left(\omega Y^{H}\right)-X^{H}\left(\omega\left(I^{D} Y^{H}\right)\right)-\omega\left(\nabla_{I^{D} X^{H}}^{H} Y^{H}-I^{D}\left(\nabla_{X^{H}}^{H} Y^{H}\right)\right)$

$$
=X^{H}\left(\omega\left(Y^{H}\right)\right)-X^{H}\left(\omega\left(Y^{H}\right)\right)-\omega\left(\nabla_{X^{H}}^{H} Y^{H}-I^{D}\left(\nabla_{X} Y\right)^{H}\right)=-\omega\left(\left(\nabla_{X} Y\right)^{H}-\left(\nabla_{X} Y\right)^{H}\right)=0
$$

(ii) $\left(\psi_{\mathrm{I}^{\mathrm{D}}} \omega\right)\left(\mathrm{X}^{\mathrm{H}}, \theta^{\mathrm{V}}\right)=\left(\psi_{I^{D} X^{H}} \omega\right) \theta^{V}=I^{D} X^{H}\left(\omega\left(\theta^{V}\right)\right)-X^{H}\left(\omega\left(I^{D} \theta^{V}\right)\right)-\omega\left(\nabla_{I^{D} X^{H}}^{H} \theta^{V}-I^{D}\left(\nabla_{X^{H}}^{H} \theta^{V}\right)\right.$

$$
\begin{aligned}
& =X^{H}\left(\omega\left(\theta^{V}\right)\right)+X^{H}\left(\omega\left(\theta^{V}\right)\right)-\omega\left(\nabla_{X^{H}}^{H} \theta^{V}-I^{D}\left(\nabla_{X} \theta\right)^{V}\right)=2 X^{H}\left(\omega\left(\theta^{V}\right)\right)-\omega\left(\left(\nabla_{X} \theta\right)^{V}+\left(\nabla_{X} \theta\right)^{V}\right) \\
& =2 X^{H}\left(\omega\left(\theta^{V}\right)\right)-2 \omega\left(\nabla_{X} \theta\right)^{V}
\end{aligned}
$$

(iii) $\left(\psi_{\mathrm{I}^{\mathrm{D}}} \omega\right)\left(\theta^{\mathrm{V}}, \mathrm{Y}^{\mathrm{H}}\right)=\left(\psi_{I^{D} \theta^{V}} \omega\right) Y^{H}=I^{D} \theta^{V}\left(\omega\left(Y^{H}\right)\right)-\theta^{V}\left(\omega\left(I^{D} Y^{H}\right)\right)-\omega\left(\nabla_{I^{D} \theta^{V}}^{H} Y^{H}-I^{D}\left(\nabla_{\theta^{V}}^{H} Y^{H}\right)\right)$

$$
=-\theta^{V}\left(\omega\left(Y^{H}\right)\right)-\theta^{V}\left(\omega\left(Y^{H}\right)\right)-\omega\left(\nabla_{\theta^{V}}^{H} Y^{H}-I^{D}\left(\nabla_{\theta^{V}}^{H} Y^{H}\right)\right)=-2 \theta^{V}\left(\omega\left(Y^{H}\right)\right)
$$

(iv) $\left(\psi_{\mathrm{I}^{\mathrm{D}}} \omega\right)\left(\theta^{\mathrm{V}}, \xi^{\mathrm{V}}\right)=\left(\psi_{I^{D} \theta^{V}} \omega\right) \xi^{V}=I^{D} \theta^{V}\left(\omega\left(\xi^{V}\right)\right)-\theta^{V}\left(\omega\left(I^{D} \xi^{V}\right)\right)-\omega\left(\nabla_{I^{D} \theta^{V}}^{H} \xi^{V}-I^{D}\left(\nabla_{\theta^{V}}^{H} \xi^{V}\right)\right)$

$$
=-\theta^{V}\left(\omega\left(\xi^{V}\right)\right)+\theta^{V}\left(\omega\left(\xi^{V}\right)\right)-\omega\left(-\nabla_{\theta^{V}}^{H} \xi^{V}-I^{D}\left(\nabla_{\theta^{V}}^{H} \xi^{V}\right)\right)=\omega \nabla_{\theta^{V}}^{H} \xi^{V}+I^{D}\left(\nabla_{\theta^{V}}^{H} \xi^{V}\right)=0
$$

Theorem 5. For an almost paracomplex structure $I^{D} \in \mathfrak{I}_{1}^{1}\left(T^{*}\left(M^{n}\right)\right)$ deffined by (11), the results of the following covariant

derivatives of $I^{D}$ with respect to vertical, complete and horizontal lifts is zero.

(i) $\left(\nabla_{X^{H}}^{H} I^{D}\right) Y^{H}=0$,

(ii) $\left(\nabla_{X^{H}}^{H} I^{D}\right) \theta^{V}=0$,

(iii) $\left(\nabla_{X^{C}}^{C} I^{D}\right) \omega^{V}=0$,

(iv) $\left(\nabla_{\omega^{V}}^{H} I^{D}\right) Y^{H}=0$,

(v) $\left(\nabla_{\omega^{V}}^{H} I^{D}\right) \theta^{V}=0$,

(vi) $\left(\nabla_{\omega^{V}}^{C} I^{D}\right) \theta^{V}=0$,

where the horizontal lift $\nabla^{H}$ of an affine connection $\nabla$ in $M^{n}$ to $T^{*}\left(M^{n}\right), X^{C}, X^{H} \in \mathfrak{I}_{0}^{1}\left(T^{*}\left(M^{n}\right)\right)$ of $X \in \mathfrak{I}_{0}^{1}\left(M^{n}\right)$ and the vertical lift $\theta^{V}, \omega^{V} \in \mathfrak{I}_{0}^{1}\left(T^{*}\left(M^{n}\right)\right)$ of $\theta, \omega \in \mathfrak{I}_{1}^{0}\left(M^{n}\right)$. 
Proof. From definition 2, proposition 1, proposition 2, (11) and (12), we get

(i) $\left(\nabla_{X^{H}}^{H} I^{D}\right) Y^{H}=\nabla_{X^{H}}^{H} I^{D} Y^{H}-I^{D}\left(\nabla_{X^{H}}^{H} Y^{H}\right)=\nabla_{X^{H}}^{H} Y^{H}-I^{D}\left(\nabla_{X} Y\right)^{H}=\left(\nabla_{X} Y\right)^{H}-\left(\nabla_{X} Y\right)^{H}=0$,

(ii) $\left(\nabla_{X^{H}}^{H} I^{D}\right) \theta^{V}=\nabla_{X^{H}}^{H} I^{D} \theta^{V}-I^{D}\left(\nabla_{X^{H}}^{H} \theta^{V}\right)=\nabla_{X^{H}} \theta^{V}-I^{D}\left(\nabla_{X} \theta\right)^{V}=-\nabla_{X} \theta^{V}+\left(\nabla_{X} \theta\right)^{V}=0$,

(iii) $\left(\nabla_{X^{C}}^{C} I^{D}\right) \omega^{V}=\nabla_{X^{C}}^{C} I^{D} \omega^{V}-I^{D} \nabla_{X^{C}}^{C} \omega^{V}=-\nabla_{X^{C}}^{C} \omega^{V}-I^{D}\left(\nabla_{X} \omega\right)^{V}=-\left(\nabla_{X} \omega\right)^{V}+\left(\nabla_{X} \omega\right)^{V}=0$,

(iv) $\left(\nabla_{\omega^{V}}^{H} I^{D}\right) Y^{H}=\nabla_{\omega^{V}}^{H} I^{D} Y^{H}-I^{D} \nabla_{\omega^{V}}^{H} Y^{H}=\nabla_{\omega^{V}}^{H} Y^{H}-I^{D} \nabla_{\omega^{V}}^{H} Y^{H}=0$,

(v) $\left(\nabla_{\omega^{V}}^{H} I^{D}\right) \theta^{V}=\nabla_{\omega^{V}}^{H} I^{D} \theta^{V}-I^{D} \nabla_{\omega^{V}} \theta^{V}=-\nabla_{\omega^{V}}^{H} \theta^{V}-I^{D} \nabla_{\omega^{V}}^{H} \theta^{V}=0$,

(vi) $\left(\nabla_{\omega^{V}}^{C} I^{D}\right) \theta^{V}=\nabla_{\omega^{V}}^{C} I^{D} \theta^{V}-I^{D} \nabla_{\omega^{V}}^{C} \theta^{V}=-\nabla_{\omega^{V}}^{C} \theta^{V}-I^{D} \nabla_{\omega^{V}}^{C} \theta^{V}=0$.

\section{Conclusion}

The paper deals with Tachibana operators, Vishnevskii operators applied to lifts with respect to almost paracomplex structure $I^{D}$ (The diagonal lift $I^{D}$ ) and covariant derivatives of almost paracomplex structure $I^{D}$ on cotangent bundle $T^{*}\left(M^{n}\right)$. Firstly, the Tachibana operators were applied to $1-$ form, vertical, complete and horizontal lifts with respect to almost paracomplex structure $I^{D}$ (The diagonal lift $I^{D}$ ) on cotangent bundle. Secondly, the Vishnevskii operators were applied to 1 -form according to the diagonal lift $I^{D}$ on cotangent bundle. Finally, we obtain several new results about covariant derivatives of almost paracomplex structure $I^{D}$ with respect to vertical, complete and horizontal lifts on $T^{*}(M)$.

\section{References}

[1] M.A.Akyol, B.Şahin, Conformal anti-invariant submersions from almost Hermitian manifolds, Turkish Journal of Mathematics, 40 (2016) 43-70.

[2] D.E.Blair, Contact Manifolds in Riemannian Geometry, Lecture Notes in Math, 509, Springer Verlag, New York, (1976).

[3] S.Büyükkütük, İ.Kişi, V.N.Mishra, G.Öztürk, Some Characterizations of Curves in Galilean 3-Space $G_{3}$, Facta Universitatis, Series: Mathematics and Informatics, 31 (2) (2016) 503-512.

[4] H.Çayır, Some Notes on Lifts of Almost Paracontact Structures, American Review of Mathematics and Statistics, 3 (1) (2015) $52-60$.

[5] H.Çayır, Lie derivatives of almost contact structure and almost paracontact structure with respect to $X^{V}$ and $X^{H}$ on tangent bundle $T(M)$, Proceedings of the Institute of Mathematics and Mechanics, National Academy of Sciences of Azerbaijan, 42 (1) (2016) 38-49.

[6] H.Çayır, Tachibana and Vishnevskii Operators Applied to $X^{V}$ and $X^{C}$ in Almost Paracontact Structure on Tangent Bundle $T(M)$, Ordu Üniversitesi Bilim ve Teknoloji Dergisi, Ordu Üniversitesi, 6 (1) (2016) 67-82.

[7] H.Çayır, Tachibana and Vishnevskii Operators Applied to $X^{V}$ and $X^{H}$ in Almost Paracontact Structure on Tangent Bundle $T(M)$, New Trends in Mathematical Sciences, 4 (3) (2016) 105-115.

[8] H.Çayır, G.Köseoğlu, Lie Derivatives of Almost Contact Structure and Almost Paracontact Structure With Respect to $X^{C}$ and $X^{V}$ on Tangent Bundle $T(M)$. New Trends in Mathematical Sciences, 4 (1) (2016) 153-159.

[9] Deepmala, L.N.Mishra, Differential operators over modules and rings as a path to the generalized differential geometry, Facta Universitatis (NIS) Ser. Math. Inform., 30 (5) (2015) 753-764.

[10] A.Gezer, L.Bilen, A.Çakmak, Properties of Modified Riemannian Extensions, Journal of Mathematical Physics, Analysis, Geometry, 11 (2) (2015) 159-173.

[11] Y.Gündüzalp, Neutral slant submanifolds of a para-Kahler manifold, Abstract and Applied Analysis, (2013), Doi:10.1155/2013/752650, pp.1-8.

[12] S. Kaneyuki, F.L.Williams, Almost Para-Contact and Para-Hodge Structures on Manifolds, Nagoya Math. J., 99 (1985) $173-187$.

[13] S.Kızıltuğ, S.Yurttançıkmaz, A.Çakmak, Normal and Rectifying Curves in the Equiform Differential Geometry of $G_{3}$, Poincare Journal of Analysis \& Applications, 2 (2014), 55 - 61 
[14] İ.Kişi, S.Büyükkütük, Deepmala, G.Öztürk, AW(k)-type Curves According to Parallel Transport Frame in Euclidean Space $E^{4}$, Facta Universitatis (NIS) Ser. Math. Inform., 31 (4) (2016).

[15] P.Libermann, Sur les Structures Presque Para-Complexes, C.R. Acad. Sci. Paris Ser. I Math., 234 (1952) 2517-2519.

[16] S.Das, Lovejoy, Fiberings on almost r-contact manifolds, Publicationes Mathematicae, Debrecen, Hungary 43 (1993) $161-167$.

[17] V.N.Mishra, Some Problems on Approximations of Functions in Banach Spaces, Ph.D.Thesis, Indian Institue of Technology, Roorkee 247 667, Uttarakhand, India (2007).

[18] V.N.Mishra, K.Khatri, L.N.Mishra, Deepmala, Inverse result in simultaneous approximation by Baskakov-Durrmeyer-Stancu operators, Journal of Inequalites and Applications, 586 (2013), doi:10.1186/1029-242X-2013-586.

[19] F.Ocak, A.A.Salimov, Geometry of the cotangent bundle with Sasakian metricsand its applications, Proc. Indian Acad. Sci. (Math. Sci.), 124 (3) (August 2014) 427-436.

[20] V.Oproiu, Some remarkable structures and connexions, defined on the tangent bundle, Rendiconti di Matematica 3 (1973) 6 VI.

[21] T.Omran, A.Sharffuddin, S.I.Husain, Lift of Structures on Manifolds, Publications de 1'Institut Mathemat1qe, Nouvelle serie, 360 (50) (1984) 93 - 97.

[22] A.A.Salimov, Tensor Operators and Their applications, Nova Science Publ., New York (2013).

[23] A.A.Salimov, H.Çayır, Some Notes On Almost Paracontact Structures, Comptes Rendus de 1'Acedemie Bulgare Des Sciences, 66 (3) (2013) 331-338.

[24] S.Sasaki, On The Differantial Geometry of Tangent Boundles of Riemannian Manifolds, Tohoku Math. J., no.10(1958) 338-358.

[25] B.Şahin, M.A.Akyol, Golden maps betwen Golden Riemannian manifolds and constancy of certain maps, Math. Commun., 19 (2014) 333-342.

[26] Vandana, Deepmala, K.Drachal, V.N.Mishra, Some algebro-geometric aspects of spacetime c-boundary, Mathematica Aeterna, accepted on July 28, in press.

[27] K.Yano, S.Ishihara, Tangent and Cotangent Bundles, Marcel Dekker Inc, New York (1973). 